Erschienen in: Dinge als Herausforderung : Kontexte, Umgangsweisen und Umwertungen von Objekten / Hahn, Hans Peter; Neumann, Friedemann (Hrsg.). - Bielefeld : transcript, 2018. (Edition Kulturwissenschaft ; 182). - S. 143-166. - ISBN 978-3-8376-4513-2 https://dx.doi.org/10.14361/9783839445136-008

\title{
Das Bildnis des General Aung San
}

\author{
Felix Girke
}

Aung San's name today evokes images of freedom, democracy, and civil rights to the people of Burma, and it is to his image that many turn during the nation's difficult hours.

(Naw 2001: 218)

At once stable and portable, images look back at us, and in doing so shed light on the meaning of absence. The visible and the invisible, seen and imagined, constitute each other through endless feedback.

(Bartmanski/Alexander 2012: 6)

\section{JULI 2015: MARTYRS' DAY IN YANGON (MYANMAR)}

In den grauen Morgenstunden des 19. Juli 2015 traf ich mich mit zwei ad-hoc eingestellten Forschungsassistentinnen vor dem Hauptquartier der »National

1 Ich danke Judith Beyer für eine kritische Durchsicht des Textes und Lisa Huurdeman für handwerkliche Hilfe. Diskussionen im Rahmen des Panels $47 »$ Dinge, die provozieren; Dinge, die vermitteln« (DGV-Tagung 2015 in Marburg) sowie auf der Konferenz »Myanmarforschung 2015« in Zürich haben mir geholfen, mein Argument zu fokussieren. 


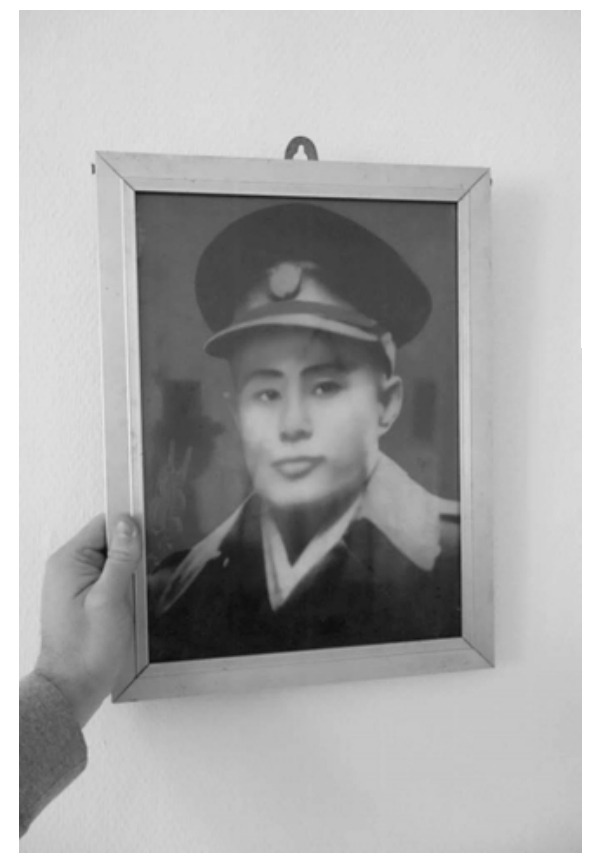

Abbildung 1: Gerahmtes Aung

San Portrait

Quelle: Sammlung Felix Girke

League of Democracy« (NLD) im Stadtviertel Bahan in Yangon, der früheren Hauptstadt Myanmars. Die beiden MA-Studentinnen der Ethnologie waren mir vom Department of Anthropology der Yangon University vermittelt worden. Ich beabsichtigte, über den ganzen 19. Juli den nationalen Feiertag »Martyrs’ Day« an den einschlägigen Schauplätzen der Metropole zu beforschen. Eine der Assistentinnen wurde von ihren Eltern begleitet, die mich in Augenschein nahmen möglicherweise war ihnen auch der Ort und die Uhrzeit unseres Treffens suspekt. Nach einem kurzen Austausch lächelten sie und überreichten mir eine mitgebrachte Gabe: ein gerahmtes, 40x30cm großes Portrait von Major-General (»Bogyoke«) Aung San, dem wichtigsten der Märtyrer, denen an diesem Tag gedacht wurde.

Bei einem Arbeitsplan, der vorsah, von 4 Uhr morgens bis zum Einbruch der Dunkelheit mit ständigen Ortswechseln der Dynamik des Gedenkens zu folgen, war dieses Objekt natürlich zunächst unpraktisch. Doch kann dieser Akt des Gebens, der meine Forschung an diesem »Martyrs’ Day« eröffnete, als ein Schlüsselmoment für eine weitergehende Analyse betrachtet werden: gerahmte Aung 
San-Portraits gehören zu den Dingen, die in Myanmar (früher Burma, im Deutschen auch »Birma $\aleph^{2}$ ) im 20. Jahrhundert und möglicherweise darüber hinaus mit das größte Potential zur Provokation trugen - und das nicht nur am Martyrs' Day. Nach einem kurzen biographischen und historischen Überblick von Kolonialzeit bis zur Periode nach 1988, als große Aufstände das Land erschütterten, diskutiere ich das Bildnis des General Aung San als säkulare Ikone, die durch ihre Materialität als gerahmte Photographie und ihrer grenzüberschreitenden Bewegung zwischen privaten Räumen und der >Straße ২ eine besondere Dynamik generieren kann.

\section{WER WAR AUNG SAN?}

Wenige Menschen schieden mit 32 Jahren aus dem Leben und hinterließen derartige Fußstapfen wie Aung San. Mehrere englischsprachige (z.B. Maung Maung 1962, Naw 2001, Silverstein 1993) und zahllose myanmarische Veröffentlichung haben sich seinem Leben und seinem Wirken gewidmet; ich nenne nur die wichtigsten Eckpunkte.

Geboren 1915 noch unter britisch-kolonialer Herrschaft im ländlichen Burma, politisierte er sich schnell, als er seine Studien an der Rangoon University aufnahm. Zusammen mit Sinnesgenossen agitierte er gegen die britischen Herrscher und deren Bildungspolitik, die letztlich nur auf die Ausbildung einer einheimischen, Empire-konformen Bürokratenkaste abzielte, und stieg zu einem landesweit anerkannten Anführer der Studentenschaft auf. In den späten 1930ern trat er der Dobama Asiayone bei (etwa: »Wir Burmesen-Assoziation«), einer anti-kolonialen Gruppierung, deren Mitglieder sich gegenseitig den Titel thakin verliehen, »Meister/Herr«, eine Anrede, die bisher exklusiv von den Briten eingefordert wurde. »Thakin Aung San« orientierte sich politisch an Sozialismus und Kommunismus und tat sich als Begründer und Mitglied verschiedener Parteien und Gruppierungen hervor.

Die burmesischen Nationalisten sahen im Zweiten Weltkrieg eine Chance, die ersehnte Unabhängigkeit und Herauslösung aus dem Empire zu erlangen, und

2 Die internationale Bezeichnung »Myanmar« wurde 1989 offiziell festgelegt; damit einher ging eine Umbenennung vieler Orte im Land. Ich verwende Burma für die Zeit bis 1988, ab dann Myanmar, respektive Rangoon und Yangon. Die überwiegend buddhistische Bevölkerungsmehrheit bezeichne ich als »Bamar«. Ein guter Sammelbegriff für die Bewohner Myanmars ist weder im Deutschen noch im Englischen etabliert, daher verwende ich den eigentlich veralteten Begriff »Burmesen «. 
Abbildung 2: Aung San-Literatur im Buchladen

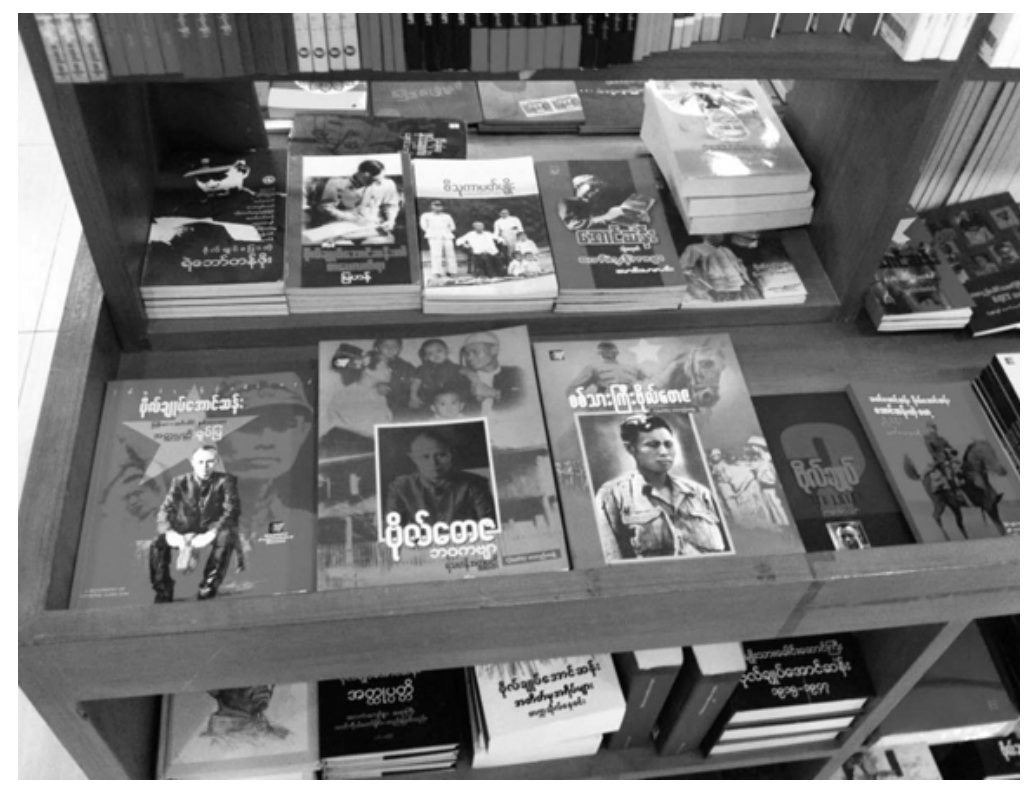

Quelle: Felix Girke

Aung San, bereits persona non grata, schmuggelte sich mit Weggefährten aus dem Land, um in China Verbündete zu finden. Von japanischen Agenten abgefangen und rekrutiert, wurde er paramilitärisch ausgebildet und kehrte nach Burma zurück, wo er mit seinen »30 Kameraden« den geplanten japanischen Angriff auf das Land mit einheimischen Korps unterstützte. Die Briten wurden 1942 aus Burma vertrieben, und die verbliebenen Truppen und Verwaltungsteile zogen sich nach Indien zurück. Die Japaner bauten in Folge parallele japanische und burmesische Administrationen auf und entließen Burma 1943 formal (aber letztlich nur vorgeblich) in die Unabhängigkeit. Aung San, in Tokyo mit Orden behängt, wurde Kriegsminister. Die Enttäuschung und Frustration der Burmesen wuchs jedoch schnell, da die Bedingungen unter den Japanern keineswegs besser als unter den Briten waren. Als sich im pazifischen Theater das Blatt schließlich zu wenden begann und die Briten aus Indien erneute Vorstöße nach Burma machten, wandte sich Aung San gegen die japanischen Machthaber. In Kooperation mit britischen Offiziellen begann er, die Vertreibung der Japaner vorzubereiten. Ein breiter Aufstand im März 1945, von ihm mitorganisiert, trug zur Niederlage der Japaner bei, und im Oktober 1945 herrschte wieder eine zivile Regierung in Rangoon. Aung 
San sollte erst einen hohen Rang in der neu gegründeten »Burma Army« bekleiden, sah seine Zukunft selber aber im zivilen-politischen Bereich. Als Präsident der AFPFL (Anti-Fascist People's Freedom League) führte er diverse politische Kräfte, konnte aber die Abspaltung der CPB (Communist Party of Burma), die er mitbegründet hatte, nicht verhindern. Die burmesischen Nationalisten strebten weiterhin v.a. nach Unabhängigkeit, und Aung San unterzeichnete im Januar 1947 in London das Aung San-Attlee Agreement, das vorsah, das Burma selber über seine Zukunft innerhalb oder außerhalb des britischen Commonwealth entscheiden können sollte. ${ }^{3}$ Eine Vorbedingung, die den Verhandlungsführern - in erster Linie ethnische Bamar - von den Briten auferlegt wurde, war, mit den ethnischen Minderheiten des Landes (in den »Frontier Areas«) zu einer Einigung zu kommen. Die Briten waren besorgt, dass die Karen (heute auch Kayin), Kachin, Chin, Shan etc. unter einer von Bamar dominierten Regierung aus dem »Ministerial Burma« benachteiligt werden könnten: Angehörige der Minderheiten, großenteils Christen, hatten von der Kolonialzeit stärker profitiert als die Bamar, und hatten auch im Laufe des Krieges mit den Briten gegen die Japaner und ihre Bamar-Landleute gekämpft.

Wieder nach Burma zurückgekehrt, besuchte Aung San die so genannte Panglong-Konferenz, auf der Vertreter von einigen ethnischen Minderheiten über ihre zukünftige Haltung zum Land debattierten. Er schaffte es kraft seiner Ehrlichkeit und Aufrichtigkeit, das Vertrauen der anwesenden Delegierten zu gewinnen. Diese bekundeten schließlich mit ihrer Unterschrift unter dem Panglong Agreement, mit der Union of Burma kooperieren zu wollen, um schnellstmöglich Unabhängigkeit für alle innerhalb der Landesgrenzen zu erreichen. Aung Sans Zusicherungen von wirtschaftlicher Kooperation, interner Autonomie und gleicher Rechte für die Bewohner der »Frontier Areas« hatten sie überzeugt.

Wenige Monate später, am 19. Juli 1947, wurde Aung San mit dem halben Kabinett seiner Schattenregierung im »Secretariat«, dem wichtigsten Regierungsgebäude in Rangoon, von den Handlangern eines Rivalen ermordet. U Saw, ein älterer Politiker, der gegen das Aung San-Attlee Agreement gestimmt hatte und der sich erhoffte, seinen früheren Einfluss wieder zu gewinnen, wurde als Urheber

3 Reproduziert hier: https://burmastar1010.files.wordpress.com/2011/06/44172419aungsan-atlee-agreement.pdf. Donnison (1970: Kapitel 8) bietet einen nachvollziehbaren Überblick der Machtkämpfe in dieser Zeit, sowohl zwischen burmesischen Politikern und britischer Verwaltung als auch in Bezug auf die Rivalitäten unter Burmesen. 
des Komplotts ausgemacht. Aus Aung San und den anderen wurden »die Märtyrer«, symbolisch gefallen bei den letzten Schritten zur Unabhängigkeit. ${ }^{4}$

Aung San war, wie eingangs erwähnt, zu diesem Zeitpunkt 32 Jahre alt und auf dem besten Weg, Premierminister zu werden. Neben seiner bewegten öffentlichen Karriere heiratete er die Krankenschwester Khin Kyi, die ihn im Rangoon General Hospital gepflegt hatte. Von ihren Kindern ist die Tochter Suu Kyi (oft auch »Aung San Suu Kyi«) heute als Friedensnobelpreisträgerin und Gesicht des Widerstands gegen das Militärregime, das jahrzehntelang das Land kontrollierte und global isolierte, weltbekannt. Sie führt seit 1988 die NLD an.

Aung Sans Verlust erschütterte das Land. Zahlreiche Autoren, die seine Ermordung diskutieren, werfen die Frage auf, was es bedeutet hätte, wenn er weiter die Zügel des Staates gehalten hätte. Hätte der quasi mit der Unabhängigkeit einsetzende Bürgerkrieg mit seinen vielen Fronten, der im Prinzip bis heute andauert, verhindert werden können? Hätte eine echte Annäherung zwischen Bamar und den ethnischen Minderheiten des Landes stattgefunden? Wäre der desaströse »burmesische Weg zum Sozialismus« ab den 1960er Jahren nicht eingeschlagen worden, der die Wirtschaft des Landes, das unter dem zweiten Weltkrieg sehr gelitten hatte, endgültig ruinierte?

Doch durch den Zeitpunkt seines Todes konnte Aung Sans Erbe in gewisser Hinsicht rein und unbefleckt bleiben, geprägt durch seine persönlichen Qualitäten und Erfolge und nicht durch Kompromisse und Realpolitik korrumpiert.

»Burmans have said that 〉Everything Aung San touched turned to gold«: perhaps this was most true in that, in the hour of his triumph, he was struck down, to become a martyr. Aung San has the glory of independence: he has no responsibility for the miseries of the civil war that followed.« (Tinker 1967: 28, vgl. Brigadier Maung Maung 2011: 70f)

\section{DIE ORTE UND FESTE DES GENERAL AUNG SAN}

Aung San war die Schlüsselfigur der burmesischen Unabhängigkeit. Er war »student leader«, ein bis heute anerkannter Status in Myanmar; er war als thakin und

4 Eigentlich wurden neun Menschen ermordet. Im Laufe der Jahrzehnte wurden Ohn Maung, der lediglich Staatssekretär in spe war, und Ko Htwe, ein junger schiitischer Leibwächter eines sunnitischen Ministers, gelegentlich nicht im selben Ausmaß gewürdigt wie die ,richtigen' Minister. Noch heute wird ihnen bei den jährlichen Gedenkfeiern als letzten der neun Märtyrer gedacht. 
Mitglied der Dobama Asiayone federführender Nationalist; er agitierte für Sozialismus und Kommunismus; er war als Guerilla und Revolutionär erst gegen die Briten und dann gegen die Japaner ein militärischer Held, der gleich zwei Kolonialmächte `besiegt hat; er war als Gast in London und Delhi ein weltweit anerkannter Sprecher für die burmesische Sache; er zeigte in Panglong die Möglichkeit einer Annäherung zwischen Bamar und Minderheiten auf; und er wurde zum nie korrumpierten (hypothetisch unkorrumpierbaren) Märtyrer. Posthum hinterließ er mit seiner Tochter Suu Kyi, so stellte sich letztlich heraus, dem im 20. Jahrhundert so schwer geplagten Land ein Versprechen auf eine bessere Zukunft. Diese Liste deutet an, wie er verschiedensten Anliegen als Projektionsfläche dienen konnte und kann.

Schon bald wurde seiner Rolle im Land auch angesichts - und man kann annehmen: wegen - seiner körperlichen Abwesenheit materiell Rechnung getragen: alleine in Yangon nimmt heute eine ganze Reihe von Orten direkten Bezug auf Aung San. Eine der wichtigsten Achsen der Downtown ist heute die Bogyoke Aung San Road. Dort liegt auch der bedeutsame Bogyoke Aung San Market. Sein altes Wohnhaus, heute ein Museum, liegt (ebenso wie die deutsche Botschaft) an der Bogyoke Aung San Museum Road, die von der Nat Mauk Road abgeht, wiederum eine vielbefahrene Ost-West-Achse etwas nördlich der Innenstadt - Nat Mauk im mittleren Burma war sein Geburtsort. Vom Museum fußläufig erreichbar ist die Aung San-Statue. ${ }^{5}$ Das zentrale Stadion in Yangon ist das Aung San-Stadium. Um die bedeutendste Pagode des ganzen Landes, die Shwedagon-Pagode in Yangon, schlängelt sich die »Märtyrer-Straße« (Arzarni Road). Diese führt zum »Martyrs' Memorial«, in dem die Überreste von Aung San und den meisten anderen Ermordeten ruhen. Im Restaurant »House of Memories« ist seine Schreibstube aus der Zeit der japanischen Besatzung erhalten. In einem College auf dem Campus der Yangon University findet sich auch noch sein Zimmer aus studentischen Tagen. Schließlich, das Secretariat: nach dem Umzug der Regierung Myanmars nach Naypyidaw vor etwa zehn Jahren wurde dieser riesige Komplex mehr oder weniger aufgegeben, und blieb der Öffentlichkeit verschlossen - der Sitzungssaal, in dem die Märtyrer starben, wurde jedoch weiterhin als Schrein gepflegt. Ein Vorort der Stadt, Aung San Myo, wurde nach dem Krieg für Sozialwohnungen für

5 In den meisten Städte Myanmars steht eine Aung San-Statue, und Aung San-Straßen sind typischerweise wichtige Boulevards. 
Veteranen bereitgestellt. Aung San wurde auf Geldscheine und auf Briefmarken gedruckt. $^{6}$

Nicht nur der Martyrs' Day, sondern auch andere säkulare Feiertage des Landes stehen in Zusammenhang mit Aung San: ${ }^{7}$ Der »Independence Day« am 4. Januar ist im Prinzip durch die Abwesenheit Aung Sans gekennzeichnet, der ihn zwar erzwungen hatte, aber nicht mehr erleben durfte. Der »Union Day« am 12. Februar markiert die Unterzeichnung des »Panglong Agreements«. Der Aufstand gegen die Japaner am 27. März 1945 war der Anlass für den »Resistance Day«, und wurde später in »Armed Forces Day« umbenannt. Aung Sans Geburtstag ist zwar kein offizieller Feiertag, wird aber oft individuell oder informell zum Gedenken genutzt: viele Freiwillige verteilen an diesem Tag eine einfache Linsenspeise mit paratha-Brot, eine spärliche Mahlzeit, die angeblich das Lieblingsgericht des auch deswegen als asketisch verstandenen Bogyoke gewesen sein soll. ${ }^{8}$

Es sei festgehalten, dass das Gedenken an Aung San öffentlich wie privat gepflegt wurde, dass es einen reichen Schatz an Gedichten, Liedgut, und oral tradierten Narrativen gab und gibt, und dass sich Wahrheit und Mythos hierbei kaum trennen lassen - wenn das denn jemand wollte.

Folglich fungierte »Aung San« als eine Projektionsfläche für Hoffnungen und Erwartungen, für Werte und Haltungen. Semiotisch betrachtet ist er jedoch weniger ein »leerer Signifikant«, der einer Beliebigkeit zuträglich ist, sondern vielmehr völlig überdeterminiert durch ein Bündel an historisch zwar zusammenhängender aber gelegentlich in Spannungsverhältnissen stehender Bezüge. Das materielle

6 Die allererste Briefmarke des unabhängigen Landes kopierte die letzte koloniale Briefmarke bis ins Detail - nur der Kopf von King George VI wurde durch den von Aung San ersetzt (Min Sun Min 2007: 40).

7 Eine Ausnahme ist der »Peasants' Day« am 2. März, der zwar offiziell den Bauern zugedacht ist, aber tatsächlich die Machtergreifung von General Ne Win 1962 markiert. Der »National Day« am zehnten Tag nach dem Vollmond des Monats Tazaungmone (gegen Ende des Jahres) erinnert an den großen studentischen Streik gegen die britischen Herrscher 1920 - zwar ohne Aung San, doch über den Akteur »Studenten« besteht auch hier eine Beziehung: Ereignisse wie dieses politisierten die Studenten, und brachten Aung San und seine Kommilitonen mittelbar hervor.

8 Im Bogyoke Aung San-Museum findet sich auf dem Esstisch im Erdgeschoss eine reproduzierte handschriftliche Notiz Aung Sans, in der er seine Frau um dieses Gericht bittet. Diese Evidenz korreliert mit Anekdoten über sein asketisches Leben, wie dem Vorfall als er befahl, die Curries der Offiziere (besser) und der einfachen Soldaten (schlechter) zusammenzugießen, um angesichts geteilter Aufgaben und Gefahren ein aus seiner Sicht unangemessenes Privileg zu beseitigen. 
Bildnis des General Aung San wird im Folgenden als eine machtvolle (säkulare) Ikone verstanden (vgl. Alexander/Bartmanski/Giesen 2012), deren mögliche Assoziationen rundweg positiv sind. Doch aus diesen erschließt sich nicht das Potential eines Aung San-Portraits, irgendjemanden zu provozieren. Dazu muss das Jahr 1988 in den Blick genommen werden.

\section{8}

Die materielle Greifbarkeit und die Direktheit der Erfahrung von Aung Sans Vermächtnis nahm mit der Machtergreifung von General Ne Win im Jahr 1962 sogar noch zu. Ne Win, ein Weggefährte Aung Sans, vermied es, einen eigenen Personenkult zu fördern und versuchte stattdessen, durch Berufung auf Aung San Legitimität für sein Regime und das Militär zu generieren (vgl. Nakanishi 2013: 2413). Die sechsundzwanzig Jahre seiner Herrschaft hatten jedoch eine fortschreitende Präkarisierung des Landes zur Folge. Das Scheitern des 1962 eingeschlagenen »Burmese Way to Socialism« war nicht länger zu leugnen. Die Lage eskalierte im Sommer 1988. Die politischen und ökonomischen Details dieser Zeit ordne ich hier einem scheinbaren Epiphänomen unter: Die Demonstranten, Menschen aus allen Teilen der Gesellschaft (vgl. Lintner 1990: 114), trugen bei Demonstrationen verlässlich ein Porträt von Aung San vorneweg (vgl. Prager 1998: 369f). Zahlreiche Beobachter haben dies registriert; ich referiere hier das einschlägige Buch Outrage des schwedischen Journalisten Bertil Lintner, um diese Verwendung des Bildnisses des Generals Aung San besser nachzuvollziehen. Schon das Titelbild meiner Ausgabe des Buchs zeigt Demonstranten mit drei Aung San-Portraits.

»We held a big meeting on the Prome Road campus [north of the city center] on June 21st', remembered Soe Win, a medical student. >Thousands of people were there and suddenly someone got the idea that we should march down town to the main Institute of Medicine in central Rangoon, where another meeting was being held. We marched off at 1 PM, a solid column of several thousand students. We took our peacock and student union flags and someone went inside the teachers' office [and] brought out Aung San's portrait to be carried in front of the demonstration.« (Lintner 1990: 75)

»At first, there were only twelve of us in a teashop in Yankin. We had gathered old precoup Union of Burma flags, student flags with the fighting peacock and portraits of Aung San which we had collected from government offices and people's homes.« (1990: 95) 
Eine Seite später reproduziert Lintner einen Bericht der Associated Press, der die Pfauenflaggen und (»1940s national hero«) Aung San-Portraits bestätigt. Ein »westlicher Diplomat« wird wie folgt zitiert:

»There were disciplined bursts of automatic riflefire into the crowds and snipers picked out the people holding flags and portraits of Aung San.« (1990: 100)

Lintners Narrativ geht unerbittlich weiter:

»The slaughter began soon afterwards. The first to be hit were a young Buddhist novice and an 18-year old girl. She was still tightly holding a portrait of Aung San when she fell dead to the street.« (1990: 101)

Später, im September, gab es erneut Demonstrationen; erneut trugen Menschen Aung San durch die Straßen, und erneut gab es Berichte, die Truppen würde bei der Niederschlagung besonders auf diejenigen zielen »who were carrying peacock flags and portraits of Aung San« (Lintner 1990: 134). Berühmt ist bis heute der Fall der jungen Win Maw Oo; Lintner berichtet, dass das sechzehnjährige Mädchen mit Schusswunden in ein Krankenhaus gebracht wurde und zitiert ihren Bruder, dass sie dort um ein Bild von Aung San bat: »At 1 pm, she died, clasping the portrait.«(1990: 134) ${ }^{9}$

Die Diagnose, dass die Träger von Bildnissen des General Aung San besonders ins Visier genommen wurden, ist heute schwer zu validieren; durch die häufige Wiederholung hat sie aber eine affektive Realität im kollektiven Gedächtnis erlangt. Sie fügt der anerkannt brutalen Reaktion der Staatsgewalt dabei noch eine psychologische Note besonderer Perfidie hinzu, und dient damit als just so-Story,

9 Ein Bild von Win Maw Oo, die blutend über die Straßen getragen wurde, ging 1988 um die Welt. Der Fall ist über den Bezug zu Aung San dahingehend bemerkenswert, dass - wie verschiedene Medien nicht ohne Pathos berichteten - Win Maw Oo ihren herbeigeeilten Vater um einen letzten Dienst bat: ")Can you promise me something? she asked. Then she made her father swear not to perform the last rites for her ,until you get the democracy we asked for'. Then she died.« (Marshall 2012) Die Eltern respektierten diesen Wunsch nach Medienberichten: Das Zeremoniell wurde am 6. Dezember 2015, wenige Wochen nach den ersten freien landesweiten Wahlen seit langer Zeit (bei denen Aung San Suu Kyis NLD 79\% der möglichen Sitze gewann), endlich nachgeholt (Min Zin 2016). 
um die Abwendung der Armee von Aung Sans Vermächtnis jenseits aller Zweifel $\mathrm{zu}$ belegen. ${ }^{10}$

Doch noch im August betrat Suu Kyi die Bühne, die lange im Ausland lebte und eher zufällig zur Pflege ihrer Mutter in Yangon war, und die doch schon immer eine vererbte Verantwortung für sihr Land empfunden hatte.

»Unintentionally, she herself came to play an important role in the future. While the uprising was gathering force she had stayed neutral, but after the August massacre pressure was building up that she should take an active role in resolving this crisis. Thousands of protesters had carried portraits of her father in demonstrations all over the country; his name was almost mythical and symbolised all that Burma was not but should be - free, democratic and prosperous. Aung San Suu Kyi continued the legacy of her father and her appearance on the political stage in Burma in August was met with excitement and high expectations." (Lintner 1990: 109)

Am 25. August hält Suu Kyi dann ihre erste öffentliche Rede an der ShwedagonPagode, einem der heiligsten Orte der Stadt und des ganzen Landes: »A huge portrait of her father, Aung San, had been placed above the stage alongside a resistance flag from World War Two.« (Lintner 1990: 115) In ihrer Rede bezieht sich Suu Kyi direkt auf das Vermächtnis ihres Vaters; als dessen Tochter habe sie nicht gleichgültig bleiben können.

") We were all surprised`, Aung Win said. >Not only did she look like her father, she spoke like him also: short, concise and right to the point.« (Lintner 1990: 116)

Lintners Bezug auf die Symbolik Aung Sans führe ich im nächsten Abschnitt aus. Festzuhalten sind hier aber zunächst die Hinweise auf die Materialität und die praktische Nutzung des Aung San-Bildnisses. In einer eleganten Formulierung weist Popham darauf hin, dass es für die Demonstranten »in absence of anyone of flesh and blood to follow « (2012: 38) eine Platzhalterfunktion hatte. ${ }^{11}$ Ein Teil der

10 Popham reproduziert sogar die Explikation eines Demonstranten, dass »I thought they could not shoot me if I was carrying a picture of General Aung San« (2012: 44). Derartige Klarheit der Informanten vereinfacht natürlich die Arbeit des Analysten. Vgl. Zöllner (2012: 292-93).

11 Die Proteste in den Straßen waren nicht zentral gesteuert, wenn auch eine Vielzahl an mehr oder weniger prominenten Politikern versuchte, in diesem Rahmen Einfluss auszuüben bzw. zu gewinnen. Siehe Belting (2012) zum Verhältnis von Bild und abwesendem Leib. 
Mythologie um Aung San speist sich aus seiner Rolle als Anführer bei öffentlichen Protesten, als Studentenführer gegen die Briten und auch später, seine öffentliche Positionierung bei Reden, Streiks und Demonstrationen.

»Those students most active in encouraging the protests saw Aung San as having been a student leader in his days and a rebellious young person fearlessly questioning authority and quite insubordinate to the illegitimate foreign invaders of Burma.« (Houtman 1999: 27)

Zudem war das Portrait schlichtweg stets greifbar, da es in allen Amtsstuben und vielen Wohnzimmern hing - womit die Verfügbarkeit von Aung San-Portraits zu den Zwecken der Demonstranten hinreichend erklärt ist. ${ }^{12}$ Doch bis dahin bestand darin keine Provokation: Der Held der Unabhängigkeit blickte hinab von der Wand als wohlwollender Märtyrer, der an das Beste in jedem gemahnt. Auf der Straße jedoch wurde Aung San zur personalisierten Verkörperung von Widerstand und zum Maßstab, an dem sich jeder Politiker, Militär und Ideologe zu messen hatte - und den natürlich qua Aung Sans unvergleichlicher Biographie niemand erreichen konnte. Aung San durch die Straßen zu tragen war daher ein unabweisbarer Vorwurf. Das seine Tochter, die ihm so ähnlich sah, ${ }^{13}$ sich nun auch gegen die Regierung und die Militärs als Alternative anbot und die Abwesenheit ihres Vaters zu füllen drohte, trug zur weiteren Eskalation bei.

\section{DAMNATIO MEMORIAE?}

Kulturhistorisch wurde die nun einsetzende Dynamik mit zwei einschlägigen Begriffen eingefangen: Gustaaf Houtman identifizierte eine »Aung San Amnesia« (1999: 15, 26-27); Susanne Prager nannte es »Ent-Aung-Sanisierung« (1998: 373). Beide diagnostizieren einen Moment, in dem sich etwas verschob: Zwar wurden bereits 1987 die ersten Banknoten ohne Aung Sans Antlitz herausgegeben, doch die Tage des Märtyrers als Ikone des Staatsapparats waren bald vorbei: »Appropriation of Aung San as icon of the opposition, in turn, seems to have served

12 So auch Tinker: »...whose portrait is in every government office and in many Burmese homes.« (Tinker 1967: 27). Doch die alten Fahnen, Jahrzehnte nach ihrer letzten Verwendung so zahlreich zu haben, werfen Fragen auf, die separat zu klären wären.

13 Siehe auch Larkin (2006: 163-64) für eine Schilderung der Dynamik und der besagten Ähnlichkeit: »[S]he has the same high cheekbones and captivating almond-shaped eyes.« Zöllner (2012: 312-16) analysiert den »Nexus« zwischen den Bildern von Vater und Tochter. 
to further hasten his marginalisation by the regime«, so Houtman (1999: 27). In den Jahren 1988 und v.a. 1989 folgten weitere Maßnahmen. Der Martyrs’ Day wurde offiziell kaum noch begangen, Staatsmedien reduzierten ihre üblichen Verweise auf den Nationalhelden, und die zuvor regelmäßigen Zeitungskolumnen über sein Wirken wurden seltener oder verschwanden ganz. Manche Politiker kritisierten Aung San sogar explizit in ihren Reden. Doch der Geist Aung Sans war nicht so leicht auszutreiben. Er konnte sowohl von der Armee als auch von seiner Tochter und deren Partei als »Vater« beansprucht werden; und dass Aung San nun missachtet wurde, um Aung San Suu Kyi als »die Tochter« zu delegitimieren (vgl. Zöllner/Ebbighausen 2015), schwächte eher die eigene Legitimität der Aung Sanvergessen(d)en Militärregierung.

Dieser partielle Ikonoklasmus ging zudem über Geld und Briefmarken und offizielle Positionierungen hinaus: in den folgenden Jahren, als Suu Kyi zur profiliertesten Oppositionsfigur geworden war und lange unter Hausarrest stand, stellten sowohl ihr bald weltberühmtes Counterfeit als auch das ihres Vaters im öffentlichen Raum Myanmar provokante und gefährliche Positionierungen dar. Touristen, die subversiv in das damals weitreichend boykottierte Land reisen wollten, brachten als gut gemeinte Solidarisierung T-Shirts mit dem Bild der Friedensnobelpreisträgerin mit. Doch trotz der Risiken für Einheimische, durch Zurschaustellung Suu Kyis oder Aung Sans vor den Augen der Sicherheitsbehörden auffällig zu werden, war das Bildnis des General Aung San nicht völlig verschwunden - die öffentliche Ent-Aung San-isierung ging mit einem stillen Widerstand einher: Privatpersonen behielten Aung San-Bildnisse in nichtöffentlichen Räumen, und Suu Kyi bemühte sich weiterhin, »das politische `Erbeく ihres Vater aus dem mythischen Begründungszusammenhang der tatmadaw [der Streitkräfte] abzulösen [...], um ihn in den Dienst der Demokratiebewegung stellen zu können « (Prager 1998: 369-70).

Die oben erwähnten Orte des Generals Aung San wurden in dieser Zeit geschlossen, gesperrt, unter Verdacht gestellt und generell gezielt ihrer vorgesehenen Nutzung entzogen: Noch bei meinem ersten Besuch in Myanmar im Jahr 2009 traf ich auf geschlossene Museen, vergitterte Gedenkstätten, vernachlässigte Mausoleen, und überwachte Parks. Als ich gegen Ende 2012 zum ersten Mal das Arzarni Gone, das »Martyrs' Memorial« nahe der Shwedagon Pagode betreten konnte - dessen Öffnung auch für Ausländer bereits ein Zeichen des einsetzenden Wandels darstellte - war es wie ausgestorben. Nur ein junger Mann zog seine Kreise um mich, und zunächst nahm ich an (geprägt durch das Misstrauen, das aus den 
Abbildung 3: Aung San und Tochter. Memorabilia, erhälttich im NLD Headquarter

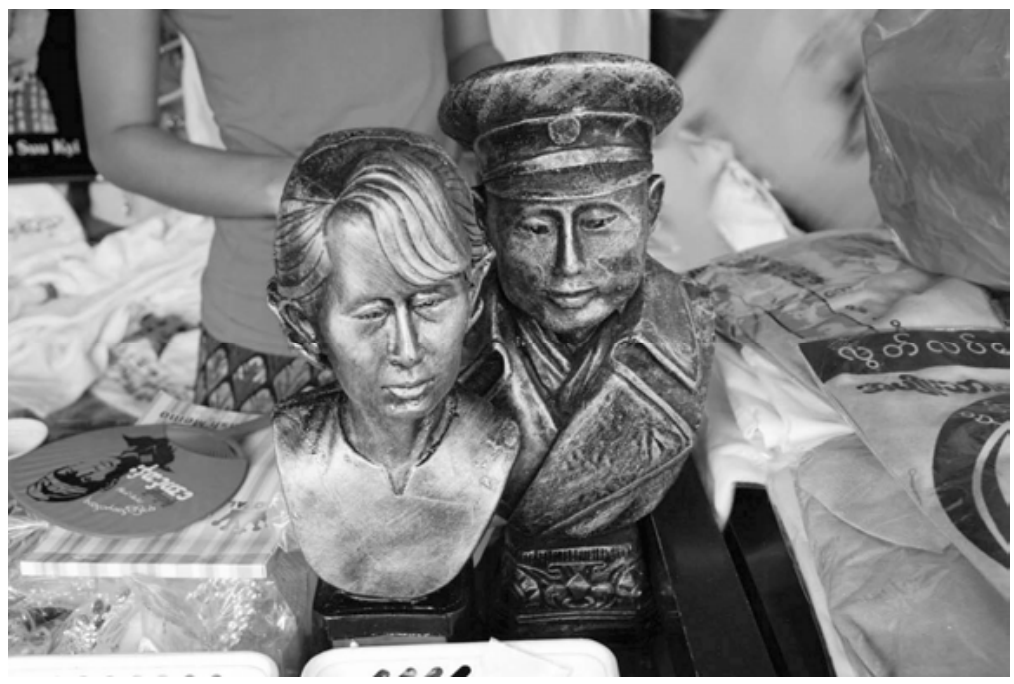

Quelle: Felix Girke

Ethnographien und Reiseberichten der Zeit sprach), dass er eine Überwachungsaufgabe erfüllte. Das Gegenteil war der Fall: Er kam näher, überreichte mir wortlos eine alte, ungültige Banknote mit Aung Sans Antlitz, und entfernte sich schleunig.

Die Militärregierung hat seit 2008 Schritt für Schritt den Weg für ein Mehrparteiensystem und eine zivile Regierung freigemacht. Die NLD konnte schon bald wieder frei im öffentlichen Raum agieren, und Aung San Suu Kyi wurde sehr bald erneut die Hoffnungsträgerin für positiven Wandel, sowohl im Land wie auBerhalb. Seither hat das damnatio memoriae seine Insistenz geradezu dramatisch verloren: Bilder von Aung San (und natürlich seiner Tochter) sind allgegenwärtig und die öffentlich-alltägliche Zurschaustellung des einst provokanten Bildes irritiert niemanden mehr.

Doch das Bildnis des General Aung San ist nicht völlig frei: als ich eines Tages im Selbstversuch nach Aung San-Memorabilia suchte (ich begann passenderweise im zentralen Bogyoke Aung San Market in der Downtown von Yangon) wurde ich nur spärlich fündig: hier ein T-Shirt, da ein Pin - bis mir schließlich ein Händler empfahl: »Go to Bahan.« Bahan, ein township von Yangon etwas nördlich der 
Innenstadt, stand hier als metonymischer Verweis auf das dort gelegene Hauptquartier der NLD, wo es in der Tat ein sehr breites Sortiment an Bildnissen und Trägermedien gab.

Die National League of Democracy hat die nur langsam abflauende Aung SanAmnesie staatlicher Stellen gut genutzt, und bedient geschickt die ungebrochene oder sogar zunehmende Nachfrage. ${ }^{14}$ Von einem Aung San-Monopol der NLD zu sprechen ginge zu weit, da mittlerweile sehr viele unabhängige Akteure mit dem Bildnis hantieren: Künstler arbeiten damit, das Internet bietet leichten Zugriff, zahllose Bücher über Aung San sind auf dem Markt. Auch die Deutungshoheit über sein Vermächtnis liegt nicht allein bei der Partei seiner Tochter. Die proaktive Besetzung dieser Nische durch die NLD verweist jedoch darauf, dass die entscheidende Rolle von Materialität für symbolische Repräsentation verstanden wird. Öffentliche Gedenkpraktiken in Myanmar - ebenso wie an anderen Orten kommen nicht ohne Dinge und Orte aus: Die Ikone ist erst eine Ikone, wenn sie gerahmt ist, oder, abgeschwächt, auf T-Shirts und Kappen und Schlüsselanhängern prangt.

Nicht ohne Grund erlaubten staatliche Stelle sogar schon vor dem Wahlsieg der NLD 2015 wieder Gedenkveranstaltungen für Aung San, und lockerten das Bilderverbot schleichend: dadurch, dass der Bogyoke wieder für kultische Handlungen der Bevölkerung (vgl. Belting 2004) verfügbar wurde, wurde der Anspruch von Suu Kyu und der NLD auf sein Erbe aufgeweicht.

\section{EINE SÄKULARE IKONE}

Es gibt natürlich mehr als nur ein Bildnis des General Aung San - ganze Bildbände sammeln die noch so körnigsten Fotos aus den 1940er Jahren, auch wenn der genaue Kontext geschweige denn Ort, Zeit, und Rechteinhaber selten festgehalten sind.

Das hier in den Fokus gerückte Portrait ist jedoch vergleichsweise historisch gut verankert. Es ist ein Ausschnitt einer größeren Abbildung, die wiederum zu einer ganzen Bilderserie gehört. Das Original zeigt Aung San in seinem britischen Militärmantel und mit großer Offizierskappe, wie er am 27.01.1947 nach Unterzeichnung des Aung San-Atlee-Abkommens vor No. 10 Downing Street auf dem

14 Anlässlich konkreter Feiertage (siehe oben) finden sich in der ganzen Stadt fliegende Händler mit Aung San-Artikeln; die NLD war jedoch die einzige Institution, die davon unabhängig dieses Material dauerhaft anbietet. 
Abbildung 4: Lokal produzierte Bildbände zu Aung San

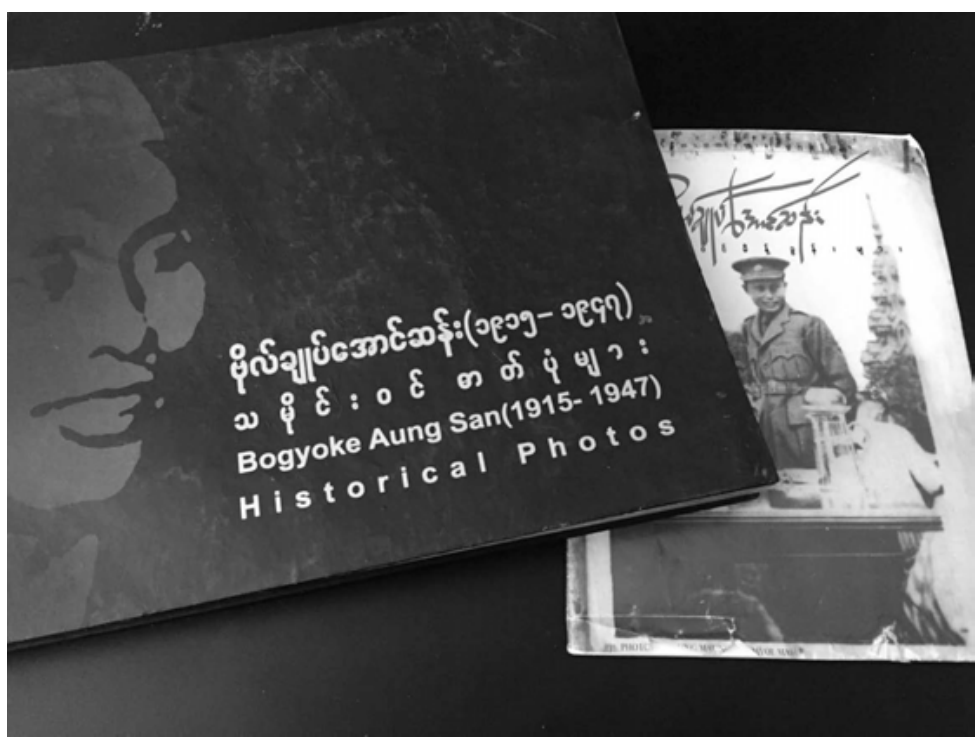

Quelle: Felix Girke

Trottoir steht. Er hat die Hände in den Taschen und blickt mit leicht schräg gehaltenem Kopf etwas von oben direkt in die Kamera. Einer seiner Mundwinkel scheint sich zu einem Lächeln zu kräuseln; ansonsten wirkt sein Gesicht außerordentlich gleichmütig. ${ }^{15}$ Warum auch nicht? Er hatte gerade in der Höhle des britischen Löwen für sein Land Historisches erreicht und sich dabei gegen interne Widersacher durchgesetzt. Viel weist darauf hin, dass es dieses spezifische Motiv war, das bis 1988 in den meisten Amtsstuben hing und deswegen eine besondere historische Rolle innehat.

Eine weitergehende Deutung dieses Bildes selber muss hier zugunsten einer kurzen theoretischen Debatte über Repräsentation ausbleiben: Was bedeutet es, das Bildnis des Generals Aung San als eine säkulare Ikone zu bezeichnen?

Der Begriff der Ikone ist alltagssprachlich allgegenwärtig, und auch Aung San Suu Kyi selber wurde bald als "globalen (Friedens-)Ikone« bezeichnet. Ich

15 Wie eine Google-Suche illustriert, ist es heute oft retuschiert (v.a. mit Weichzeichner) und als Teil von Montagen zu finden. Es wäre eine interessante weiterführende Übung, anhand von Fokusgruppen zu eruieren, wie Bürger Myanmars die verschiedenen Bildnisse Aung Sans affektiv und historisch vergleichen bzw. bewerten. 
möchte jedoch auf den dinglichen Aspekt des Bildes zurückkommen, wie er von religiösen Ikonen wie in der christlichen Orthodoxie vertraut ist. Dies ist genau der Punkt, wo die bisherige Literatur zu Aung San meines Erachtens zu kurz greift. Als historische Figur ist er überdeterminiert - durch seine politische Bedeutung, seine eigenen Texte und deren Exegese über 70 Jahre, durch Vereinnahmung durch Zeitgenossen und Epigonen, durch seine Verflechtung mit verschiedenen Parteien und Bewegungen. Doch alles Gedenken (und sei es als strategische Instrumentalisierung) ist auch immer soziale Handlung, die körperlich vollzogen werden muss und auffällig auf Dinge und Orte zurückgreift.

Der Kultursoziologe Werner Binder hat die Kernaspakte von säkularen Ikonen herausgearbeitet. Für ihn liegt jenseits der visuellen Oberfläche die eigentliche ikonische Tiefe (2012: 102). In Abgrenzung zum Kunsthistoriker Panofsky (welcher letztlich eindeutige Interpretationen von Ikonen postuliert) betont Binder, dass diese emergente Tiefe in der Interaktion des Betrachtenden mit der Oberfläche erst entsteht - somit besteht auf beiden Seiten eine gewisse Autonomie, und die Erfahrung eines Bildes kann nie vollständig verbal wiedergegeben werden. Säkulare Ikonen unterscheiden sich von religiösen Ikonen für ihn eher in ihrem Bezug als in ihrer Funktion: beide sind kollektive Symbole, die ähnlich affektiv erlebt werden - säkulare Ikonen sind wie im Falle der burmesischen Märtyrer »almost exclusively images that show human rights violations and war crimes « (2012: 104). Durch ihre Verschmelzung von Referenz (hier: Aung San) und Transzendenz (die Abstraktionen, für die er stehen kann, das Versprechen, das er hinterließ) liegt die Kraft säkularer Ikonen darin, abstrakte Vorstellungen wie Menschenwürde konkret erscheinen zu lassen (2012: 106). Binder wandelt explizit auf Durkheims Spuren, der argumentierte, dass sich »die kollektiven Gefühle ihrer selbst nur bewußt werden können, indem sie sich an äußere Objekte anheften« (Durkheim 2007: 614).

Auf den konkreten Fall bezogen liegt es nahe zu folgern, dass die grundlegende Sakralität aller Ikonen letztlich die Dichotomie zwischen religiös und säkular überlagert. Säkularität wäre hier eher als ein ethnologisches Problem zu sehen - wie kann man in einem Land wie Myanmar, in dem Gedenkformen und ein großer Teil der figürlich-bildenden Kunst überhaupt in höfischer und monastischer Praxis entstanden sind, überhaupt Säkularität isolieren?

Aung San kommt auch hierbei eine Sonderrolle zu, weil er der erste neuzeitliche Nicht-Mönch und Nicht-König war, der derartige ikonische Bedeutung erlangt hat. Es gab schlichtweg keine einheimischen Präzedenzfälle, an denen sich das Gedenken an Aung San und der Umgang mit seinem Bildnis hätten anlehnen 
Abbildung 5: Gedenkpraktiken am Fuße der Aung San-Statue, Yangon, 19. Juli 2015

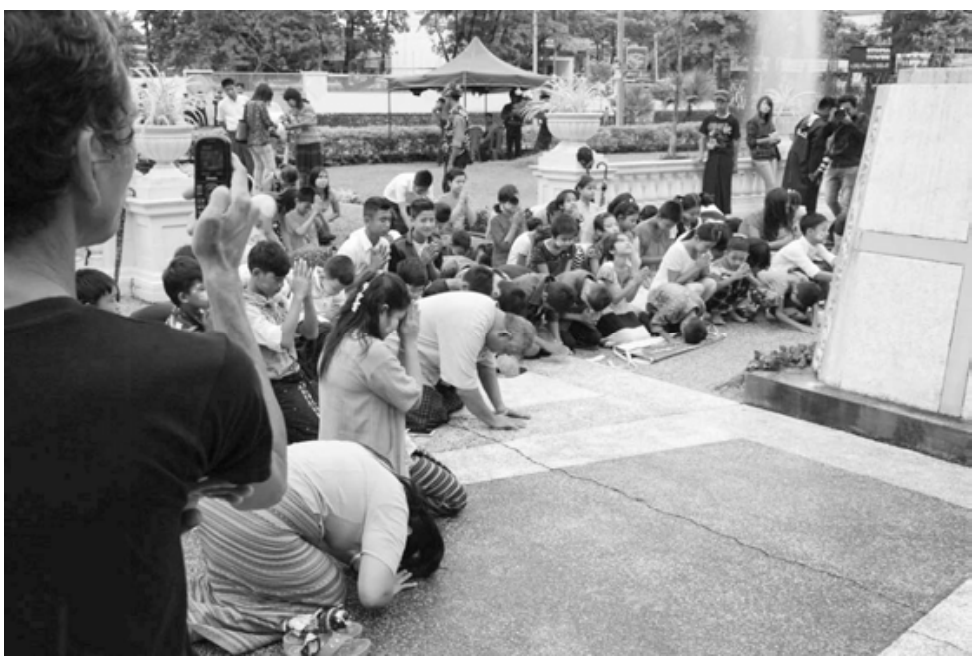

Quelle: Felix Girke

können. ${ }^{16}$ Diese Feststellung, verbunden mit den Unwägbarkeiten des 20. Jahrhunderts in Myanmar, mahnt an, dass die Ikonenanalyse vorläufig bleiben muss, um mit dynamischen Änderungen einer emergenten Praxis umgehen zu können und eben nicht eine finale Deutung eines Bildes liefert.

Die Macht des Bildes liegt also in der Interaktion zwischen Blick und ikonischer Tiefe. In Verweis auf die frühere »Ära des Bildes« (im Gegensatz zur heutigen »Ära der Kunst«) betont Belting, das ikonische Bilder »mit der Aura des Sakralen aus der Dingwelt herausrag[t]en, der sie andererseits doch angehörten« (2004: 17). Die Abbildung von menschlichen Abwesenden (Toten) entwickelt ihre efferveszente Eigendynamik: »Man möchte verehren, was man sichtbar vor Augen hat: Aber das kann keine Erzählung sein, sondern nur eine Person. Die Bilder enthalten ein Moment der Erzählung, auch wenn sie keine Erzählung sind.« (2004: 20) Dies entspricht der oben erwähnten ikonische Tiefe - das Bild zieht den Betrachter sprichwörtlich in seinen Bann, einen evokativen Bann der Erinnerung und

16 Die Debatte über das Verhältnis von Aung San und dem burmesischen Königtum, v.a. die milleniaristische Vorstellung des minlaung, des kommenden Königs bzw. Kronanwärters, findet sich am besten bei Prager (1998). 
Vorstellungskraft anregt. Doch die Kraft dieses Banns und die spezifische Wirkung seines Bildnisses sind bei Aung San stark kontextabhängig.

\section{EIN BILDNIS ZWISCHEN PRIVATSPHÄRE UND ÖFFENTLICHKEIT}

Aung San ist vieles für viele. Sein Bild ist Provokation, Vermittlung, Hoffnung; Symbol für eine bessere Zeit, eine gerechte, wenn auch unter einem Führer. Dem gleichmütigen Blick des Bogyoke hält kein falscher Schein und kein (behördliches) Unrecht stand. Beim Umgang mit dem Bild im Haus wie auch auf der Straße scheint mir ein chiasmisches Verhältnis zu bestehen: In Anlehnung an das einleitende Zitat von Angelene Naw wenden sich zwar viele Menschen in Krisenzeiten an das Bildnis von Aung San, aber zugleich ist Krise, wenn Aung San durch die Straßen getragen wird. Spätestens post-1988 ist das Herumtragen eines Bildnisses des General Aung San eine etablierte Protestform und Provokationsweise. Dabei hängt die Provokation eng mit der Materialität zusammen; denn nimmt man dem Bild seinen öffentlichen Raum, seine Re-Präsentierbarkeit, kann es zwar im Privaten weiter existieren, doch verliert seine praktischen, performativen bzw. rhetorischen Qualitäten.

Die Frage nach dem säkularen Charakter der Ikone muss auch empirisch offen bleiben. Houtman betont den funktionalen Unterschied zwischen azani (seine Transkription von ar zar ni, »Märtyrer«) und nat: Die nats, Geisterwesen zum Bewundern oder Bedauern, die alle als Menschen noch gewaltsam zu Tode gekommen waren, wurden von der allgemeinen Öffentlichkeit verehrt und rituell milde gestimmt. Märtyrer wurden jedoch auch offiziell von Regierungen anerkannt und als Helden gefeiert (1999: 282). Steinberg wagt sich etwas später schon weiter vor: »In the popular view, [Aung San] has become almost a nat, a spirit of a powerful person who died a violent and untimely death« (2010: 42). Es wäre eine spannende Herausforderung, in Myanmar auf Spurensuche zu gehen um zu ermessen, ob Geistermedien oder andere nat-Experten tatsächlich an dem einen oder anderen Ort begonnen haben, Aung San in das Pantheon aufzunehmen. Hier muss die Feststellung genügen, dass Aung San wichtige Kriterien eines nats erfüllt. ${ }^{17}$

17 Siehe auch MacLaughlins Artikel in Foreign Policy (2015), der mit Berufung auf einen jungen Wissenschaftler aus Myanmar an der SOAS (London) diesen Schritt bereits als vollzogen ansieht: »Aung San's image at home has been elevated to that of a Nat - a 
Abbildung 6: Hinterkopf mit Aung San-Tätowierung, Yangon, 19. Juli 2015

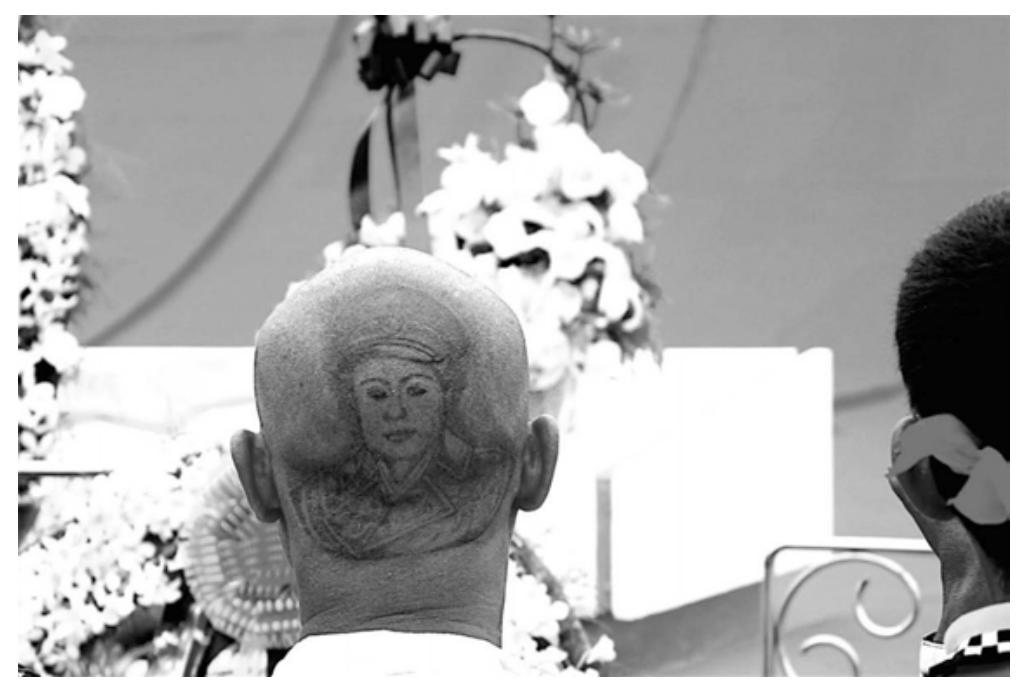

Quelle: Felix Girke

Die praktische Art und Weise, wie ihm Respekt gezollt wird, lehnt sich zudem an das in Myanmar angetroffene Geflecht von buddhistischen und animistischen Ritualen an: »paying respect«, wie es oft bezeichnet wird, kniend mit gefalteten Händen, geschlossenen Augen und Mantras auf der Lippe, kann kaum als rein säkular betrachtet werden. Der Schutz, den sich seine Träger vom Bildnis des Generals Aung San bei Demonstrationen erhofften, gemahnt zugleich an Spiros Diskussion von »apotropäischem Buddhismus « (1971: 140-161) und dessen »Krisenritualen« (1971: 255-275), die generell auf Artefakte wie Buddhabilder, Rosenkränze, und Talismane zurückgriffen. $\mathrm{Zu}$ letzterer Kategorie zählt Spiro auch die in Myanmar heute noch bzw. wieder sehr gängigen Tätowierungen. ${ }^{18}$

spirit that is traditionally worshiped in Myanmar alongside the Buddhist faith." Mir gegenüber wurde dies in Myanmar nie derartig eindeutige und absolute geäußert.

18 Neben diesen allzu materiellen Bildträgern ermöglicht das Digitale natürlich eine totale Entfesselung des Bildes. Bereits 2011, als Internetnutzung und Mobiltelefonie in Myanmar bei weitem noch nicht so verbreitet und zugänglich waren wie heute, berichtete das Irrawaddy Magazin, dass anlässlich Aung Sans 96. Geburtstag viele User sozialer Medien ihre Profilbilder mit dem Bildnis des Generals Aung San ersetzt hatten, eine zu dieser Zeit sehr innovative Verwendungsweise (Ko Htwe 2011). 
Wie oben erwähnt wirft der vorzeitige Tod Aung Sans viele Fragen über verpasste Möglichkeiten und denkbare historische Alternativpfade auf (so schon Tinker 1967: 28). Aber auch sein Gedenken ist, wie dargelegt, dynamisch und kontingent. Es scheint klare Einschränkungen zu geben: Aung San ist keine Marke, sein Bild und sein Name können nicht kommerziell verwendet werden. ${ }^{19}$ Auch wenn er nicht zum nat werden sollte, so bleibt es eine offene Frage, wie das Bildnis des Generals Aung San in Zukunft eingesetzt werden wird. Ein unter der Schirmherrschaft von Suu Kyi geplanter Film über sein Leben steckt schon so lange in der Vorbereitung, dass 2018 bereits ein Film über den angekündigten Aung San-Film erscheinen soll.

Heute scheint mir die breite und weitreichende Desillusionierung vieler Beobachter und Bürger mit Aung San Suu Kyi, die als »State Counselor« eine zentrale Rolle im Staat einnimmt und die entsprechend ihrer eigenen, bereits vor den Wahlen 2015 gemachten Ansage »über dem Präsidenten« steht ${ }^{20}$, ein wichtiger Faktor für eine weitere Beschäftigung mit dem Bildnis des General Aung San (aktuell: Kaspar 2017). Wenn entscheidende Versprechen nicht eingelöst werden, wenn Hoffnungen enttäuscht werden und der ersehnte »Wandel« ausbleibt - kann dann eines Tages Aung San auch gegen seine Tochter auf die Straße getragen werden? Trotz der häufigen Zusammen- oder Gegenüberstellung von Vater und Tochter bleibt der Märtyrer polyvalenter. Dies bewahrt dem Bildnis und der historischen Figur eine Autonomie, die davor schützt, einseitig vereinnahmt zu werden.

In dem Moment, als mir am Martyrs' Day 2015 das Aung San-Portrait in die Hand gedrückt wurde, machte ich mir wenig Gedanken über den Ursprung des Bildes oder was die Geber damit bezweckten. Mittlerweile steht es - wie viele seiner Artgenossen in Myanmar auch - in einem Wohnzimmer, an einer Art Ehrenplatz. Ich nehme nicht an, dass die Eltern meiner Assistentin erwarteten, dass

19 Auch wenn die Pressefreiheit in den letzten Jahren Fortschritte gemacht hat, besteht noch eine selten thematisierte Prüfungsbehörde für jede Art öffentlicher Werbung. Als ich das Kassenpersonal im Aung San-Museum darauf ansprach, wie auffallend niedrig ich den Eintrittspreis für Ausländer fände (der an anderen Museen in der Regel ein Vielfaches des Eintrittspreises für Burmesen beträgt), wurde mir direkt gesagt, mit Bogyoke Aung San solle kein Profit erzielt werden.

20 Hier das sofort als problematisch eingestufte Zitat im Kontext: ")If we win, and the NLD forms a government, I will be above the president. It's a very simple message <, a relaxed and smiling Suu Kyi told reporters at her lakeside home in Yangon. When asked if this arrangement violated the constitution, Suu Kyi replied: >No. The constitution says nothing about somebody being above the president.« (Marshall/Mclaughlin 2015). 
ich das Bildnis sichtbar mit mir führen würde, um selber an den Gedenkfeiern eine aktive Rolle einzunehmen. Aber sie gaben mir ein vieldeutiges Ding, das provozieren kann und zugleich ein uneingelöstes Versprechen von Hoffnung für Myanmar birgt - ein Bildnis, dass nicht altert, auch wenn die Realität hässlicher wird. Die Spannung zwischen dem öffentlichen und dem privaten Umgang mit dem Bildnis des Generals Aung San eröffnet auch weiterhin einen relevanten Einblick in die gesellschaftlichen Dynamiken des Landes.

\section{LITERATUR}

Alexander, Jeffrey C./Bartmanski, Dominik/Giesen, Bernhard (2012): Iconic power. Materiality and meaning in social life, New York: Palgrave Macmillan. Bartmanski, Dominik/Alexander, Jeffrey C. (2012): »Introduction. Materiality and meaning in social life. Toward an iconic turn in cultural sociology«, in: Jeffrey C. Alexander/Dominik Bartmanski/Bernhard Giesen (Hg.), Iconic power, New York: Palgrave Macmillan, S. 1-12.

Belting, Hans (2004 [1990]): Bild und Kult. Eine Geschichte des Bildes vor dem Zeitalter der Kunst, München: C.H. Beck.

Belting, Hans (2012): »Body and image«, in: Jeffrey C. Alexander/Dominik Bartmanski/Bernhard Giesen (Hg.), Iconic power, New York: Palgrave Macmillan, S. 187-202.

Binder, Werner (2012): »The emergence of iconic depth. Secular icons in a comparative perspective«, in Jeffrey C. Alexander/Dominik Bartmanski/Bernhard Giesen (Hg.), Iconic power, New York: Palgrave Macmillan, S.101-116.

Donnison, Frank Siegfried Vernon (1970): Burma, London: Ernest Benn.

Durkheim, Émile (2007 [1981]): Die elementaren Formen des religiösen Lebens, Berlin: Insel/Verlag der Weltreligionen.

Houtman, Gustaaf (1999): Mental culture in Burmese crisis politics: Aung San Suu Kyi and the National League for Democracy, Tokyo: ILCAA.

Larkin, Emma (2006 [2004]): Finding George Orwell in Burma, London: Penguin. Lintner, Bertil (1990): Outrage. Burma's struggle for democracy, Bangkok: White Lotus.

Maung Maung (2011 [1962]): Aung San of Burma (Myanmar). Introduction by Professor Harry J. Benda, Yangon: Unity Publishing House.

Min Sun Min (2007): Stamps of Burma. A historical record through 1988, Chiang Mai: Mekong.

Nakanishi, Yoshihiro (2013): Strong soldiers, failed revolution. The state and military in Burma, 1962-88, Singapur und Kyoto: NUS Press. 
Naw, Angelene (2001): Aung San and the struggle for Burmese independence, Chiang Mai: Silkworm.

Popham, Peter (2012): The lady and the peacock. The life of Aung San Suu Kyi, New York: Experiment.

Prager, Susanne (1998): Nationalismus als kulturelle Reproduktion. Aung San und die Entstehung des postkolonialen Birma, Dissertation, Fakultät für Orientalistik und Altertumswissenschaft, Universität Heidelberg.

Silverstein, Josef (1992): The political legacy of Aung San. Revised edition. With an introductory essay. Southeast Asia Program Series 11, Ithaca: Cornell University.

Spiro, Melford E. (1971): Buddhism and society. A great tradition and its Burmese vicissitudes, London: George Allen \& Unwin

Steinberg, David I. (2010): Burma/Myanmar. What everyone needs to know, Oxford: Oxford University Press.

Tinker, Hugh (1967 [1957]): The Union of Burma. A study of the first years of independence. Fourth edition. London u.a.: Oxford University Press.

Zöllner, Hans-Bernd (2012): The beast and the beauty. The history of conflict between the military and Aung San Suu Kyi in Myanmar, 1988-2011, set in a global context, Berlin: Regiospectra; Yangon: Pansodan University.

Zöllner, Hans-Bernd/Ebbighausen, Rodion (2015): Die Tochter. Aung San Suu Kyi, Angermünde: Horlemann.

\section{ONLINEQUELLEN}

Kaspar, Andrew D. (2017): Shades of ,bad old days' in NLD-led Burma: Human Rights Watch. Democratic Voice of Burma. URL: http://www. dvb.no/news/shades-bad-old-days-nld-led-burma-human-rights-watch/73550

Ko Htwe (2011): Aung San's 96th birthday commemorated. The Irrawadddy. URL: http://www2.irrawaddy.com/article.php?art id=20748

Marshall, Andrew R.C. (2012): Girl's death 24 years ago haunts quest for justice in reformist Myanmar, Reuters. URL: http:/www.reuters.com/article/us-m yanmar-schoolgirl-idUSBRE88F03E20120916

Marshall, Andrew R.C./Timothy McLaughlin (2015): Myanmar's Suu Kyi says will be above president in new government, Reuters World News. URL: http://www.reuters.com/article/us-myanmar-election-idUSKCN0SU0AR2015 1105 
McLaughlin, Timothy (2015): Mythmaking in the New Myanmar. Foreign Policy. URL: http://foreignpolicy.com/2015/11/06/mythmaking-in-the-newmyanmar-aung-san-suu-kyi-elections-burma-movie/

Min Zin (2016): Burma's painful democratic rebirth. Foreign Policy. URL: http://foreignpolicy.com/2016/02/04/burmas-painful-democratic-rebirth/ 